\title{
Victory of the resistance
}

\author{
I Dayaram \\ Medical student, University of KwaZulu-Natal \\ Corresponding author: Ishani Dayaram (ishani.dayaram@gmail.com)
}

Alexander Fleming, in his Nobel Prize acceptance speech in 1945, foreshadowed the development of antimicrobial resistance (AMR) and the current crisis. ${ }^{1}$ It was already apparent to the man to whom we owe the discovery of penicillin at that early stage and yet it is now an impending situation where we face antibiotic pan-resistance. When future generations look back in history at the incredible opportunity we were given, it will be seen as a mere spark that could have ignited into a revolution in modern medicine. Yet due to human error and irresponsible practice, we have squandered the possibilities of eradicating many infections and the subsequent monumental impact on global healthcare. Despite years of awareness campaigns and additional surveillance systems to prevent inappropriate prescribing, we have to be pragmatic in our approach and prepare for what will happen in the post-antibiotic era.

This poses specific challenges to the field of surgery where antibiotics are used as a prophylactic measure, a cornerstone of infection prevention. Antibiotic resistance is present in every country therefore it is not a remote possibility to ready ourselves for this eventuality. ${ }^{2}$ So, this begs the question, how will surgery progress in the post-antibiotic era? To postulate answers, we must not just look towards the future but simultaneously to the past to visualise this.

Surgeries happened well before the advent of antimicrobials and will have to be performed in the future. It was understandably much more risky and infections were rampant, whether this was because germ theory was not well understood or because of substandard hygiene practices amongst other reasons. However, many of these simple measures have fallen out in favour of more technologically developed alternatives. The World Health Organisation in 2016 released 29 recommendations to prevent surgical site infections to be implemented worldwide. ${ }^{3}$ Critically, it recognised the threat of AMR and warned against the prolongation of antibiotic prophylaxis postsurgery with a view to prevent further resistance. Soon antibiotics will not be an option at all. In this time, there will have to be careful insight into sterile surgical practices. Easy measures, such as not shaving a patient prior to surgery and maintaining normal body temperature will become routine procedure. Additionally, simple solutions such as silver in its topical form or in devices would also have a place in surgical practice. Moreover, the overall wellbeing of the patient will be a greater consideration. Making sure they are well nourished and hydrated and in as good health as possible to stave off infection will become important. This may be insufficient to prevent and not enough to fight off infections, especially those that are aggressively resistant to treatments, therefore additional measures are warranted.

There are many innovations that are currently in various stages of development that need greater investment rather than developing ubiquitous antibiotics or ones that promise to treat resistant strains because mutations occur much faster than we can forge the swords against it. This means we need to find another weapon to fight infection other than antimicrobials. There is a scope for the development of immunological agents to ensure that the host is able to mount a sufficient response against any infectious attack. Whilst vaccines are a remote possibility, it is unlikely to be a long-lasting solution with the rapid rate of mutations exhibited by many microbes. In lieu of this, there should be research into finding ways to augment the immune system to halt infections in its tracks. However, most surgeries introduce foreign bodies to the body, even if it is just suture material so it is important that an overactive immune system does not fight against this and go into an excessively inflammatory state which can lead to unfortunate complications and adverse outcomes. This can be done by using materials that the body will recognise or not react to, such as using grafts from the patients themselves or transforming their tissue where possible. Minimally invasive procedures will be especially useful as they pose a much smaller risk of infection. ${ }^{4}$ Laparoscopic surgeries are already being opted for in appropriate cases and robotics is a rapidly growing field. In the face of pan-resistance, this is clearly what the future has to offer. Laser therapies and others that do not require an incision at all, such as those to break up gallstones, would be much preferable to the conventional method of treatment. Pharmacological alternatives to surgery would also be used more frequently. This would be due to the perceived higher risks that a surgical patient would face, regardless of which approach achieves better results. Radiological developments must be done in conjunction as thorough visualisation of any problem or abnormality will help prevent unnecessary surgeries and, furthermore, once the extent of the procedure is intricately mapped out, this negates the need to perform invasive procedures or expose large areas of internal organs to infectious agents.

Protocols would also be modified so that surgical patients are given greater priority. Longer recovery from surgery would need to be in a sterile environment as opposed to in 
a general ward as is currently done. This would help prevent contracting surgical site infections. However, it is important to recognise that these stringent guidelines including those on hygiene, are difficult to maintain particularly in resourceconstrained settings that have a high patient load. Therefore, the burden of surgery associated morbidity and mortality will be borne largely by low and middle income countries. This is unacceptable owing to the fact that these countries already account for a higher proportion of nosocomial infections. ${ }^{5}$ Whilst this is not a priority now, in the future this imbalance should be highlighted as a key area for attention.

AMR progressing to pan-resistance would certainly be a tragedy. However, the tenacity of surgeons cannot be overstated and despite the challenges that would be present, there would be strides of changes the discipline would undergo to ensure safety and quality care. This includes alterations in procedure and hygiene practices as well as the exciting developments to surgical technique and pharmacological advances. This would be a victory in spite of the resistance.

\section{REFERENCES}

1. Rosenblatt-Farrell N. The Landscape of Antibiotic Resistance. Environ Health Perspect. 2009;117(6):A244-A250.

2. World Health Organization. Antimicrobial Resistance Fact Sheet. Geneva: WHO, 2016 [accessed 28 September 2017]. Available from: http://www.who.int/mediacentre/factsheets/ fs 194/en/.

3. World Health Organization. Global guidelines on the prevention of surgical site infection. Geneva: WHO, 2016 [accessed 28 September 2017]. Available from: http://www.who.int/gpsc/ssiprevention-guidelines/en/.

4. Varela JE, Wilson SE, Nguyen NT Laparoscopic surgery significantly reduces surgical-site infections compared with open surgery. Surg Endosc. 2010;24(2):270-276. doi: 10.1007/ s00464-009-0569-1

5. Rosenthal VD, Maki DG, Mehta $\mathrm{Y}$, et al. International Nosocomial Infection Control Consortium (INICC) report, data summary of 43 countries for 2007-2012. Device-associated module. Am J Infect Control. 2014;42(9):942-956. doi: http:// dx.doi.org/10.1016/j.ajic.2014.05.029

\section{Author's biography}

I am currently a fourth year student at the Nelson R. Mandela School of Medicine. Both my parents are doctors which inspired a love of medicine from a young age. I have a younger brother who is studying maths. I am still undecided about which specialty to pursue as a future career simply because I enjoy so much of medicine- admittedly a welcome problem to have. I especially love being in a clinical environment. I am also passionate about research and would love to incorporate this into whatever path I choose because the impact one can have with research alone is immense. My aspirations are strongly underpinned by the belief that every person has a right to quality healthcare. In my spare time, I enjoy reading, specifically Tudor history, learning languages, travelling and spending time with friends and family.

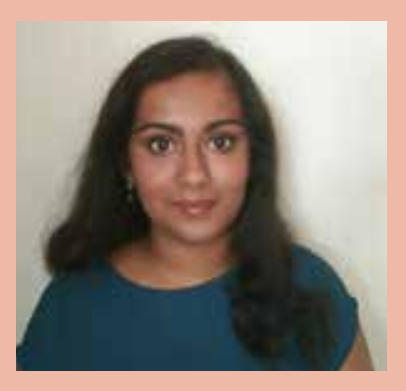

